\title{
Akupunktur bei Kreuzschmerzen: Wissen die Therapeuten, was sie tun?
}

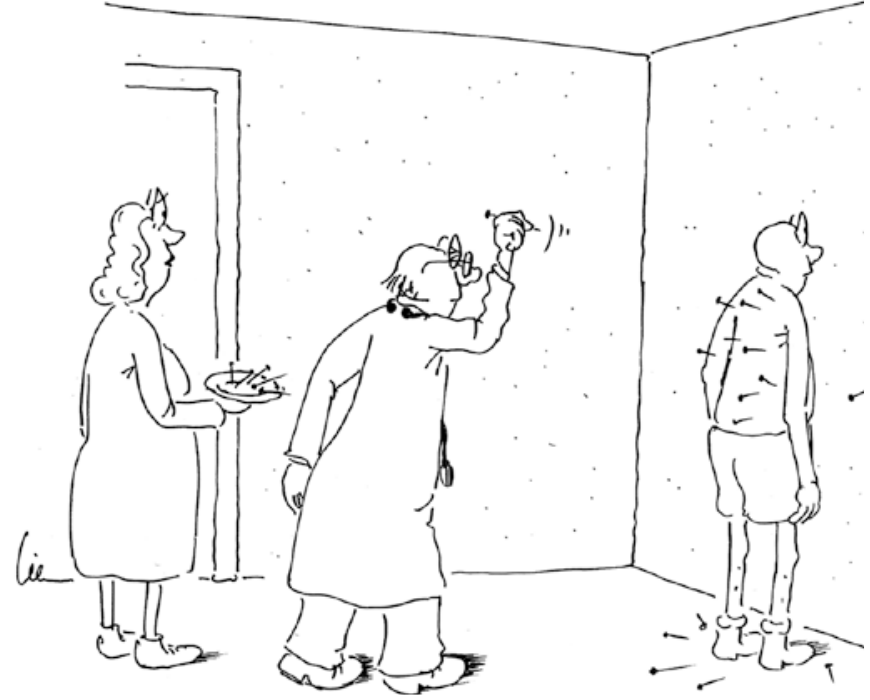

Die offiziellen britischen Leitlinien zur Behandlung unspezifischer Kreuzschmerzen empfehlen auch den Einsatz der Akupunktur. Wie Akupunkteure bei dieser Indikation genau vorgehen, ist bislang aber wenig untersucht.

- Englische Forscher baten 300 Akupunkteure, die zu drei verschiedenen Berufsgruppen gehörten, einen Fragebogen auszufüllen. Eruiert werden sollte, wie diese Therapeuten Kreuz- schmerzen behandeln. Insgesamt konnten 129 Antworten ausgewertet werden.

Die Ergebnisse zeigen, dass die Therapeuten verschiedenen Akupunkturschulen angehörten, dass sie einen Rückenpatienten meist initial ein- oder zweimal pro Woche behandelten, dass sie dabei 2-30 Nadeln pro Sitzung einsetzten, dass die Therapie 0-40 min dauerte und dass 121 verschiedene Akupunkturpunkte genadelt wurden.

Ebenso variabel waren die bei Rückenschmerzen eingesetzten Begleitbe- handlungen. So setzten 3\% niemals, $15 \%$ der Befragten immer Massage ein. Die Autoren meinen, dass diese Daten „riesige Variationen“ in der Behandlungsweise aufzeigen.

\section{Kommentar}

Vor vielen Jahren haben wir bereits gezeigt, dass selbst Akupunkturexperten sich höchst uneinig darüber sind, was eine adäquate und eine inadäquate Akupunktur gegen Rückenprobleme ist. Die vorliegende Untersuchung bestätigt diese tiefe Verwirrung. Es scheint fast so, als würde jede Art der Nadelung praktiziert. Somit ist es kaum möglich zu sagen, was eine richtige und eine falsche Therapie ist. Sogar eine völlig willkürliche Wahl von Akupunkturpunkten wäre dann vertretbar. Fazit: Der Optimist meint, dass jede Akupunktur richtig sei, und der Pessimist kann mit dem gleichen Recht behaupten, dass jede Art der Akupunktur falsch ist.

E. ERNST :

- F. L. Bishop FL, S. Zaman, G. T. Lewith Acupuncture for low back pain: a survey of clinical practice in the UK. Complement. Ther. Med. 19 (2011) 144-148

\section{Erleichtert die Aromatherapie eine Kolonoskopie?}

\section{Die Aromatherapie soll im Rahmen einer Gastroskopie angstlösend wirken. Jetzt wurde untersucht, ob dieser Effekt auch bei der Kolonoskopie zu beobachten ist.}

— Forscher aus Taiwan haben 27 Personen, die sich einer Kolonoskopie unterziehen mussten, in zwei Gruppen randomisiert. Die Verumgruppe inhalierte während des Eingriffs Neroli-Öl, dem entspannende Effekte nachgesagt werden. Die Kontrollgruppe inhalierte Sonnenblumenöl, das keine derartigen Wirkungen aufweisen soll. Als Zielgrößen dieser Studie galten die Stress- und
Schmerzempfindungen während der Kolonoskopie.

Beide Parameter zeigten keine signifikanten Differenzen zwischen den Gruppen. Dennoch meinen die Autoren, dass die Aromatherapie eine „billige, wirksame und sichere Therapie sei“"

\section{Kommentar}

Natürlich wäre es schön, wenn Aromatherapie die Unannehmlichkeiten einer Kolonoskopie oder ähnlicher Eingriffe verringern könnte. Jedoch scheint hier der Wunsch der Vater des Gedankens gewesen zu sein. Zwar zeigen einige Messgrößen wie z. B. der Blutdruck signifikante Effekte, aber die eigentlichen, patientenbezogenen Parameter sprechen nicht für die Wirksamkeit der Aromatherapie. Leider sehen wir das in alternativmedizinischen Studien nicht selten: Die Zielgrößen liefern nicht das gewünschte Ergebnis - was liegt da näher, als durch Heranziehung surrogater Endpunke ein negatives Resultat in ein positives Ergebnis umzuwandeln?

E. ERNST 